Available online at GSC Online Press Directory

GSC Biological and Pharmaceutical Sciences

e-ISSN: 2581-3250, CODEN (USA): GBPSC2

Journal homepage: https://www.gsconlinepress.com/journals/gscbps

(RESEARCH ARTICLE)

\title{
Effectiveness of different plant extracts along with new chemistry insecticide against Tribolium castaneum
} \author{
Bilal 2, Arshad Abdullah ${ }^{2}$, Ihsan Talal ${ }^{2}$ and Mushtaq Zulfiqar ${ }^{2}$ \\ 1 Department of entomology, Bahaudin Zakariya University, Bahadur Sub Campus Layyah. \\ 2 Department of Entomology, University of Agriculture Faisalabad. \\ ${ }^{3}$ Department of Zoology, Wildlife \& fisheries, University of Agriculture Faisalabad.
}

Shaker Muhammad Afsar Ali 1, ${ }^{*}$, Asif Muhammad Ammad 2, Shahzad Khalil Ahmad 3, Ikhlaq Muhammad

Publication history: Received on 19 September 2020; revised on 03 October 2020; accepted on 10 October 2020

Article DOI: https://doi.org/10.30574/gscbps.2020.13.1.0301

\begin{abstract}
Different insect pests attack stored grain products during storage. T. castaneum are the most threatening pests of stored grains. Feeding of adults and larvae is stored grains. The extracts of Datura stramonium and Zingiber officinale prepared with acetone and Deltamethrin was applied. Five concentrations 0.01\%, 0.02\%, 0.03\%, 0.04\%, 0.05\% were prepared with one controlled concentration of both the plants and the Deltamethrin. Treatments were applied on the filter paper check their possible lethal effects against T. castaneum. The data concerning mortality was recorded after time interval 24, 48 and 72 hours and analyzed statistically using ANOVA (analysis of variance) and comparison of means of treatment means was analyzed with the help of Tuckey's honestly significant difference (THSD) test. The mean mortality of Tribolium castaneum were $90 \%$ after 72 hours by exposure of Deltamethrin. The mean values of \% mortality caused by effect of Datura stramonium against T. castaneum was $52 \%$. The mean values of \% mortality caused by plant extract Zingiber officinale against T. castaneum was $41 \%$ after 72 hours. Results revealed that Deltamethrin is more effective than plant extracts. Datura stramonium is more effective than Zingiber officinale.
\end{abstract}

Keywords: Datura stramonium; Zingiber officinale; Deltamethrin and mortality

\section{Introduction}

Stored grains commodities are attacked by a variety of beetles and larvae of lepidopteron insect pets. Of them, Tribolium castaneum (Herbst) is a severe insect pest of stored commodities throughout the world. The larvae and adults feed actively on extensive range of long term commodities such as cereals, spices, beans etc. Mainly attack the germ part of seed leading to its poor germination (Mondal, 1994). Huge infestation occurs in favorable conditions (hot and humid conditions). Moreover, T. castaneum attacks in huge number, leave behind shredded skins, secrete quinines and other toxic substances (Hussain et al., 2008). This insect is widely spreading various climatic regions throughout the world (Shafique et al., 2006). Total annual post-harvest deficits of stored cereals credited to various natural factors in the storages range between 10-20\% of overall development (Phillips and Throne, 2010).

Traditional insecticides (fumigants and other synthetic insecticides) have been in practice for the stored grains insect pest management. But excess, repeated and injudicious use of these pesticides poses a lot of problems to humans and natural environment (Collins et al., 2005) use of these fumigants has results in soil contamination (Pietrantonio et al., 1999), resistance development in the insects (Phosphine resistance) in insects (Irshad et al., 1990). Indiscriminate use of organophosphates produces resistance in a number of stored grain insect pests. After that, the use of these

\footnotetext{
*Corresponding author: Shaker Muhammad Afsar Ali Email: Afsaraliagrarians@gmail.com Department of entomology, Bahaudin Zakariya University, Bahadur Sub Campus Layyah 
organophosphates was decreased (Fang et al., 2002). Therefore, there is need to develop and use those pesticides which are environment friendly, less toxic to humans and can control store grain insect pest efficiently. Due to toxic and harsh nature of the fumigants, adverse effects have occurred on human beings and natural enemies such as predator, parasites and parasitoids (Isman, 2006). So there is urgent need to move towards eco-friendly and sustainable insect pest measures to cope the insect pest's infestations problems in stored grains products.

Bio pesticides represent one of the finest used chemicals for the launching of environmental friendly and efficient control of stored commodities insect pests (Copping and Menn 2000). All plant derived substances have repellent as well as feeding deterrence properties (Isman, 2006). The insecticidal action of wide-ranging plant extracts plus more generally of some plant based products chemicals established fact and their utilize was looked after for a large number of previous time throughout all the farming parts of the planet (Regnault et al. 2012). Plant extracts can be potential and reliable sources for the effective management of stored products insect pests (Nazemi and Moharamipour, 2008). Many plants have been used in past a now a days against different insect pests species. Plant materials have repellent effects against stored products insect pests (Najafabadi et al., 2014) and ovicidal effects (Tunc, et al., 2000). Extracts of four medicinal plants were tested for their toxic effects against T. castaneum. Repellent effects of various plants have been examined against stored grains insect pests (Mishra and Tripathi, 2011). Plant extracts have growth inhibition potentials against T. castaneum (Islam et al., 2016). Use of A. indica and other plant materials have been practiced and found effective against insect pests (Isman, 2006). Oils of Ricinus communis have toxic against T. castaneum (Rehman et al., 2018). Garlic oil can be helpful for successful control of Tribolium castaneum (Herbst) (Yang et al., 2010). Repellent Artemisia vulgaris oils have been reported against Tribolium castaneum (Wang et al., 2006). Extract of Azadirachta indica and some plats was found toxic against red flour beetle (Islam and Talukder, 2005). Plant extracts were found effective against rice weevil (Saljoqi et al., 2006). Plants belong have many toxic substance and volatile compounds which can be explored for the efficient management of insect pests (Suthisut et al. 2011). Scented oils from aromatic plants have been reported to have feeding deterrence (Tripathi et al., 2001) and toxic effects can be applied as potential and sustainable alternatives to synthetic insecticides in IPM program for efficient management of stored grains insect pests. Besides these many other researchers have also documented the repellent and toxic effects of different plant materials against insects.

The discovery of new chemistry insecticides has opened new horizons in insect pest management. New chemistry insecticides are the microorganisms derived insecticides and have been applied for the management of stored grain insect pests as well as filed insect pests (Hertlein et al., 2011). These insecticides are eco-friendly, have comparatively less toxic effects and have low mammal toxicity compared to other synthetic insecticides (Magdaalena, 2009).

\subsection{Objectives of the study}

Investigation of insecticidal potential of Datura stramonium and Zingiber officinale as plant extract against T. castaneum.

To study the relative toxicity of new chemistry insecticides Deltamethrin.

\section{Material and methods}

The material included population of red flour beetle (Tribolium castaneum), Deltamethrin, Datura extracts, Ginger (Zingiber officinale), plastic jars, treated wheat, flour, sieves, incubator, rubber bands, electric grind, muslin cloth and fine hair brushes.

\subsection{Insects' collection}

Adult insects of $T$. castaneum was collected from godown, houses, grain markets for present experiment.

\subsection{Rearing of insects}

After collecting T. castaneum adult insects, the population insects were kept in jars. Flour was rearing media for $T$. castaneum. Flour and wheat was used for the better growth of insects for rearing. These jars were kept in growth chamber at $28 \pm 2{ }^{\circ} \mathrm{C}$ and $69 \pm 5 \%$ relative humidity. Adults were placed in jars for 5 days. After that adults were shifted in other jar. Eggs of adults were present in flour. Then these eggs were reared for getting F1 generation. This generation was of same age insects. Favorable conditions were provided to jars of flour. After hatching eggs, same age of adults was produced which was used for experiment. 


\subsection{Plant extracts Bioassay}

Powdered form of plant material was obtained by washing with distilled water, drying in shadow and grinding with electric grander. After that grinding was done. Extraction of plants was prepared by using solvent with plant powder. Acetone was used as solvent for extraction of plant materials with addition of $50 \mathrm{~g}$ powder of each plant powder in 250 $\mathrm{ml}$ of acetone. By using cotton plug and aluminum cover the mouth of flaks was covered, that covering prevent evaporation of acetone form flasks. Flasks were placed on rotating shaker at $215 \mathrm{rpm}$ for $24 \mathrm{~h}$. After that mixture was filtered. Whitman filter paper was used for filtration. First of all, $99.9 \%$ standard solution of four dilutions of all extracts was prepared in acetone. The experiment was planned by using Complete Randomized Design and every treatment repeated for three time. Aliquots of these concentration were applied on filter paper by using pipette. A control treatment was also used. With the help of homogeneous distribution of plant extracts was obtained. After evaporation of acetone, twenty unsexed adults of Tribolium castaneum were placed in required jar. Jars was covered with rubber band to prevent escape of insects. Mortality data of insects was taken 24, 48 and 72 hours.

\subsection{Insecticide Bioassay}

Five concentrations $0.01 \%, 0.02 \%, 0.03 \%, 0.04 \%, 0.05 \%$ was formed with one controlled concentration of Deltamethrin which was formed in $99.9 \mathrm{ml}$ distilled water from standard solution. Syringe was used for the application of concentrations with $0.3 \mathrm{ml}$ ratio. Then concentrations were passed from filter paper and dried for one night. Treated filter papers were placed in all dishes and insects T. castaneum were kept separately as 20/dish. Their data regarding mortality was collected after time intervals of 24, 48 and 72 hrs. Percent corrected mortality was calculated using subsequent Abbott's (1925) formula:

\% Corrected Mortality: M (\%)-(Mc \%)/ (100- (Mc \%)×100

Where, Mo= Observed mortality and Mc=Mortality in control unit

\subsection{Statistical analysis}

The experiment was planned by using Complete Randomized Design. Data were analyzed statistically using analysis of variance (ANOVA) and treatment means were compared with the help of Tuckey's honestly significant difference (THSD) test.

\section{Results}

Table 1 Mean \% mortality of population of Red flour beetle (T. castaneum) by the effect of insecticide Deltamethrin with different concentrations after 24, 48 and 72 hours.

\begin{tabular}{|l|l|l|l|}
\hline \multirow{2}{*}{ Concentration } & \multicolumn{4}{|l|}{ Mean Mortality \pm S. E } & 72 hours \\
\cline { 2 - 4 } & 24 hours & 48 hours & $52.72 \pm 2.29 \mathrm{~d}$ \\
\hline $0.01 \%$ & $31.03 \pm 2.18 \mathrm{c}$ & $37.57 \pm 2.11 \mathrm{~d}$ & $60.01 \pm 2.46 \mathrm{~cd}$ \\
\hline $0.02 \%$ & $39.65 \pm 2.37 \mathrm{c}$ & $48.21 \pm 2.15 \mathrm{c}$ & $69.09 \pm 2.74 \mathrm{c}$ \\
\hline $0.03 \%$ & $50.01 \pm 2.45 \mathrm{~b}$ & $60.71 \pm 2.23 \mathrm{~b}$ & $80.01 \pm 3.01 \mathrm{~b}$ \\
\hline $0.04 \%$ & $51.72 \pm 3.00 \mathrm{~b}$ & $69.65 \pm 2.57 \mathrm{ab}$ & $90.90 \pm 3.25 \mathrm{a}$ \\
\hline $0.05 \%$ & $65.51 \pm 3.08 \mathrm{a}$ & $78.50 \pm 2.98 \mathrm{a}$ & \\
\hline
\end{tabular}

This table shows that the percentage mortality caused by five exposure treatments of insecticide Deltamethrin with various concentrations (0.01\%-0.05\%) with one control group against Tribolium castaneum at 24, 48 and 72 hours' exposure period. Results revealed that mortality was increased by increasing concentration. Highest mean mortality was 90.90 occurred at $0.05 \%$ concentration after 72 hours' exposures time period and minimum mortality was 31.03 at $0.01 \%$ concentration at 24 hours' exposure period. 
Table 2 Mean \% mortality of population of T. castaneum by the effect of plant extract Datura stramonium with different concentrations at 24, 48 and 72 hours.

\begin{tabular}{|l|l|l|l|}
\hline \multirow{2}{*}{ Concentration } & \multicolumn{3}{|l|}{ Mean Mortality \pm S. E } \\
\cline { 2 - 4 } & $\mathbf{2 4}$ hours & $\mathbf{4 8}$ hours & $\mathbf{7 2}$ hours \\
\hline $0.01 \%$ & $3.45 \pm 1.72 \mathrm{c}$ & $10.72 \pm 2.11 \mathrm{c}$ & $21.82 \pm 2.69 \mathrm{c}$ \\
\hline $0.02 \%$ & $8.64 \pm 1.98 \mathrm{c}$ & $17.86 \pm 2.16 \mathrm{bc}$ & $30.91 \pm 2.78 \mathrm{bc}$ \\
\hline $0.03 \%$ & $18.96 \pm 2.11 \mathrm{~b}$ & $23.22 \pm 2.35 \mathrm{~b}$ & $36.36 \pm 3.22 \mathrm{~b}$ \\
\hline $0.04 \%$ & $25.86 \pm 2.32 \mathrm{~b}$ & $33.93 \pm 2.66 \mathrm{a}$ & $41.80 \pm 3.45 \mathrm{ab}$ \\
\hline $0.05 \%$ & $34.48 \pm 2.43 \mathrm{a}$ & $41.07 \pm 2.98 \mathrm{a}$ & $52.72 \pm 3.81 \mathrm{a}$ \\
\hline
\end{tabular}

The percentage mortality caused by five exposure treatments of insecticide Deltamethrin Datura stramonium with various concentrations (0.01\%-0.05\%) with one control group against Tribolium castaneum at 24, 48 and 72 hours' exposure period. Results revealed that mortality was increased by increasing concentration. Highest mean mortality was 52.72 occurred at $0.05 \%$ concentration after 72 hours' exposures time period and minimum mortality was 3.45 at $0.01 \%$ concentration at 24 hours' exposure period.

Table 3 Mean \% mortality of Layyah population of T. castaneum by the effect of plant extract Zingiber officinale with different concentrations at 24, 48 and 72 hours.

\begin{tabular}{|l|l|l|l|}
\hline \multirow{2}{*}{ Concentration } & \multicolumn{3}{|l|}{ Mean Mortality \pm S.E } \\
\cline { 2 - 4 } & $\mathbf{2 4}$ hours & $\mathbf{4 8}$ hours & $\mathbf{7 2}$ hours \\
\hline $0.01 \%$ & $0.01 \pm 1.7544 \mathrm{~d}$ & $0.07 \pm 1.7856 \mathrm{c}$ & $5.45 \pm 2.1512 \mathrm{~d}$ \\
\hline $0.02 \%$ & $1.74 \pm 1.8365 \mathrm{~cd}$ & $5.36 \pm 1.8832 \mathrm{bc}$ & $14.54 \pm 2.3357 \mathrm{~cd}$ \\
\hline $0.03 \%$ & $7.01 \pm 2.1145 \mathrm{bc}$ & $12.06 \pm 2.0021 \mathrm{~b}$ & $21.82 \pm 2.7172 \mathrm{bc}$ \\
\hline $0.04 \%$ & $14.03 \pm 2.3910 \mathrm{~b}$ & $21.34 \pm 2.2956 \mathrm{a}$ & $29.03 \pm 2.9913 \mathrm{~b}$ \\
\hline $0.05 \%$ & $22.80 \pm 2.4811 \mathrm{a}$ & $23.20 \pm 2.5252 \mathrm{a}$ & $41.82 \pm 3.0423 \mathrm{a}$ \\
\hline
\end{tabular}

The percentage mortality caused by five exposure treatments of insecticide Deltamethrin Zingiber officinale with various concentrations (0.01\%-0.05\%) with one control group against Tribolium castaneum at 24, 48 and 72 hours' exposure period. Results revealed that mortality was increased by increasing concentration. Highest mean mortality was 41.82 occurred at $0.05 \%$ concentration after 72 hours' exposures time period and minimum mortality was 0.01 at $0.01 \%$ concentration at 24 hours' exposure period.

\section{Conclusion}

Effect of plant extracts Datura stramonium and Zingiber officinale along with new chemistry insecticide Deltamethrin with various concentrations $0.01 \%, 0.02 \%, 0.03 \%, 0.04 \%$ and $0.05 \%$ along with one control group against T. castaneum Herbst. Table 1 showed the percentage of mortality by ANOVA of Tribolium castaneum after exposure five treatments with different concentrations of Deltamethrin (0.01\%-0.05\%) with one control group at 24 hours' exposure time. The results found were highly significant. The percentage mortality of T. castaneum at 24, 48 and 72-hour exposure period showed in table 1 . that mortality was increased by increasing concentration. Maximum mean mortality was $90 \%$ at $0.05 \%$ concentration after 72 hours and minimum mortality was 31.037 at $0.01 \%$ concentration at 24 hours' exposure period. Table 2 showed percentage of mortality of T. castaneum by the exposure of five treatments of Datura stramonium with different concentrations (0.01\%-0.05\%) at 24, 48 and 72 hours' exposure time. The results found were highly significant. The percentage mortality of T. castaneum at 72-hour exposure period showed in table 2 . Highest mean mortality was $52 \%$ at $0.05 \%$ concentration after 72 hours and minimum mortality was $3.45 \%$ at $0.01 \%$ concentration at 24 hours' exposure period. Table 3 showed the percentage of mortality by ANOVA of Tribolium castaneum after exposure of five treatments with different concentration of Zingiber officinale (0.01\%-0.05\%) along with one control 
group at 24, 48 and 72 hours' exposure time. Results revealed that mortality increased by increasing concentration. Highest mean mortality was $41.82 \%$ at $0.05 \%$ concentration after 72 hours and least mortality was $0.01 \%$ at $0.01 \%$ concentration at 24 hours' exposure period.

\section{Compliance with ethical standards}

\section{Acknowledgments}

All authors contribute equally and with all manners.

\section{Disclosure of conflict of interest}

No observed conflict of interest among the author's.

\section{References}

[1] Collins P J, Daglish G J, Pavic H, Kopittke R.A. Response of mixed-age cultures of phosphine-resistant and susceptible strains of lesser grain borer, Rhyzopertha dominica, to phosphine at a range of concentrations and exposure periods. J.Stored Prod. Res. 2005; 41(4): 373-385.

[2] Copping LG, JJ Menn. Biopesticides a review of their mode of Action and efficacy. Pest Manag. Sci. 2000; 56: 651676.

[3] Fang L, B Subramanyam, FH Arthur. Effective of spinosad on four classes of wheat against five stored products insect. J. Econ. Entomol. 2002; 95: 640-650.

[4] Hertlein MB, GD Thompson, B Subramanyam, CG Athanassiou. Spinosad: a new natural product for stored grain protection. J. Stored Prod. Res. 2011; 47: 131-146.

[5] Hussain R, Ashfaq M, Saleem MA, Ahmed SOHAIL. Toxicity of some insecticides with novel modes of action against malathion-resistant and organophosphate-susceptible strains of Tribolium castaneum larvae. International J.Agri. Bio. 2005; 7(5): 768-772.

[6] Irshad M, WA Gillani. Resistance in Tribolium castaneum (Herbst) (Coleoptera: Tenebrionidae) against malathion. Pak. J. Zool. 1990; 2(2): 257-262.

[7] Islam MS, FA Talukder. Toxic and residual effects of Azadirachta indica, Tagetes erecta and Cynodon dactylon seed extracts and leaf powders towards Tribolium castaneum. J. plant Diseases and Prot. 2005; 112(6): 594-601.

[8] Islam W, A Rasool, Z Wu. Inhibitory effects of medicinal plant extracts against Tribolium castaneum (Herbst.) (Coleoptera: Tenebrionidae). J. Agric. Sci. 2016; 3: 15-20.

[9] Isman MB. Botanical insecticides, deterrents, and repellents in modern agriculture and increasingly regulated world. Ann. Rev. Entomol. 2006; 51: 45-66.

[10] Magdaalena WM. Effect of plant essential oils on the development and fertility of grain weevil (Sitophilus granarius). J. Cent. Eur. Agri. 2009; 10: 357-360.

[11] Mishra BB, SP Tripathi. Repellent activity of plant derived essential oils against Sitophilous oryzae (Linnaeus) and Tribolium casteneum (Herbst). Singapore J. Sci. Res. 2011; 1(2): 173-178.

[12] Mondal KAMSH. Flour beetles Tribolium spp. (Coleoptera:Tenebrionidae aspects and their control. Agri, Zool. Rev. 1994; 6: 95-195.

[13] Najafabadi SSM, E Beiramizadeh, R Zarei. Repellency and toxicity of three plants leaves extraction against Oryzaephilus surinamensis L. and Tribolium astaneum Herbst. J. Biodivers. Environ. Sci. 2014; 4(6): 26-32.

[14] Nazemi RJ, S Moharamipour. Repellency of Nerium oleander L., Lavandulla officinalis L. and Ferula assafoetida L. extracts on Tribolium castaneum (Herbst). Iran. J. Med. Aromat. Plants. 2008; 23(4): 443-452.

[15] Phillips TW and JE Throne. Biorational approaches to managing stored-product insects. Annual Review Entomol. 2010; 55: 375-397.

[16] Pietrantonio PV, J Benedict. Effect of new cotton insecticide chemistries, tebufenozide, spinosad and chlorfenapyr, on Orius insidious and two Cotesia species. Southwestern Entomologist. 1999; 24(1): 21-29. 
[17] Regnault RC, C Vincent, JT Arnason. Essential oils in insect control. low-risk products in a high-stakes world. Annu. Rev. Entomol. 2012; 57: 405-424.

[18] Rehman H, M Hasan, Q Ali, M Yasir, S Saleem, S Mirza, HU Shakir, AM Alvi, HM Ahmed. Potential of three indigenous plants extracts for the control of Tribolium castaneum (Herbst) and Rhyzopertha dominica (Fab.). Pak. Entomol. 2017; 40(1): 31-37.

[19] Saljoqi AUR, MK Afridi, SA Khan, S Rehman. Effects of six plant extracts on rice weevil Sitophilus oryzae L. in the stored wheat grains. J. Agric. Biol. Sci. 2006; 1(4): 1-5.

[20] Shafique M, M Ahmad, MA Chaudry. Feeding Preference and Development of Tribolium castaneum (Herbst.) in Wheat Products. Pak. J. Zool. 2006; 38(1): 27-31.

[21] Suthisut D, PG Fields, A Chandrapatya. Fumigant toxicity of essential oils from three Thai plants (Zingiberaceae) and their major compounds against Sitophilus zeamais and Tribolium castaneum and two parasitoids. J. Stored Prod. Res. 2011; 47(3): 222-230.

[22] Tripathi AK, V Pragapati, KK Aggarwal, S Kumar. Toxicity feeding deterrence and effect of activity of 1, 8, cinecola from Artemisia unnica on progeny production of Tribolium castaneum (Coleoptera: Tenebrionidae). J. Econ. Entomol. 2001; 94: 979-983.

[23] Tunc I, BM Berger, F Erler, F Dagli. Ovicidal activity of essential oils from plants against two stored-product insects. J. Stored Prod. Res. 2000; 36: 161-168.

[24] Wang J, F Zhu, X M Zhou, C Y Niu, CL Lei. Repellent and fumigant activity of essential oil from Artemisia vulgaris to Tribolium castaneum (Herbst) (Coleoptera: Tenebrionidae). J. Stored Prod. Res. 2006; 42(3): 339-347.

[25] Yang FL, Li XG, Zhu F, Lei CL. Structural characterization of nanoparticles loaded with garlic essential oil and their insecticidal activity against Tribolium castaneum (Herbst)(Coleoptera: Tenebrionidae). J. Agric. Food Chem. 2009; 57(21): 10156-10162. 